\title{
Scalar model of effective field theory in curved space
}

\author{
Tiago G. Ribeiro ${ }^{a, b}$ and Ilya L. Shapiro ${ }^{a, c, d}$ \\ ${ }^{a}$ Departamento de Fúsica, ICE, Universidade Federal de Juiz de Fora, \\ Juiz de Fora, 36036-100, Minas Gerais, Brazil \\ ${ }^{b}$ Fundação Centro de Políticas Públicas e Avaliação da Educação (Fundação CAEd), \\ Juiz de Fora, 36038-330, Minas Gerais, Brazil \\ ${ }^{c}$ Tomsk State Pedagogical University, \\ Tomsk, 634041, Russia \\ ${ }^{d}$ Tomsk State University, \\ Tomsk, 634050, Russia \\ E-mail: tgribeiro@ice.ufjf.br, shapiro@fisica.ufjf.br
}

ABSTRACT: We consider, in more details than it was done previously, the effective lowenergy behavior in the quantum theory of a light scalar field coupled to another scalar with much larger mass. The main target of our work is an IR decoupling of heavy degrees of freedom, including in the diagrams with mixed light-heavy contents in the loops. It is shown that the one-loop diagrams with mixed internal lines produce an IR non-local contributions which are exactly the same as the ones in the theory of the light scalar alone, with the effective self-interaction which can be obtained by the functional integration of the heavy scalar, almost neglecting its kinetic term. The same effect takes place in curved space, regardless of a larger amount of non-localities which show up in the effective model.

KeYwords: Effective Field Theories, Models of Quantum Gravity

ARXIV EPRINT: 1908.01937 


\section{Contents}

1 Introduction 1

2 The model and its MS-scheme renormalization 3

2.1 Classical action 3

2.2 UV divergences 3

$2.3 \beta$-functions 4

3 Matching UV and IR at the tree level $\quad 6$

4 One-loop calculations and effective approach $\quad 8$

4.1 One-loop corrections in full theory 8

$\begin{array}{lll}4.2 & \text { Dimensional regularization } & 9\end{array}$

5 Asymptotic behavior $\quad 11$

5.1 Matching with IR at the one-loop level 11

6 Decoupling in a weak gravitational field 13

$\begin{array}{llr}7 & \text { Conclusions } & 17\end{array}$

8 Note added $\quad 18$

\section{Introduction}

The effective approach to field theory has an utmost importance at both classical and quantum levels. The main idea behind this approach is that low-energy physics (infrared, or IR) may be worked with independently on the fundamental physics at high energy scale (in the ultraviolet, or UV). For instance, in the IR the framework of effective models assumes that we do not need to account the degrees of freedom present in the high energy theories [1]. The reviews of traditional realizations of this idea in Particle Physics can be found in $[2,3]$ and the part concerning quantum gravity was extensively discussed in [4].

The standard effective approach to quantum gravity (see, e.g., [4]) is based on treating all higher derivative terms as small perturbations [5, 6]. This treatment is indeed well justified if we do not care about the underlying fundamental theory of quantum gravity that should be valid in the UV [7]. At the same time, in quantum gravity such a fundamental theory is not known. This becomes a problem if we recognize that all known approaches, including string theory, have the same level of difficulties concerning higher derivatives and ghosts $[8,9]$. Thus, it looks like we need to have to worry about ultimate quantum gravity, even if are interested only in the IR effective approaches. 
Recently, there were two new approaches to quantum gravity which deal directly with the problem of higher derivative ghosts. The first one is to construct the theory polynomial in metric derivatives (with the polynomials of the even order higher than four) and design it to be superrenormalizable [10]. One can provide that this theory has only complex conjugate unphysical states. In this case one can show that the theory has unitary $S$ matrix when quantized within the Lee-Wick approach $[11,12]$. Another possibility to avoid the problems caused by ghosts is to introduce the nonlocal structures into the classical action [13-16]. The corresponding form factors can be fine tuned to avoid the ghost states. However, it was shown in [17] that such a fine tuning is always destroyed by any type of quantum gravity or semiclassical corrections, and as a result at the quantum level in this theory there is an infinite amount of ghost-like states with complex poles.

In all known approached to the fundamental theory of quantum gravity the situation is such that the higher derivative ghosts are present, being those degrees of freedom with real poles, tachyons or some combinations of these two types. Looking from this perspective on the effective approach, one of the most important questions is what remains from the higher derivative quantum gravity in the IR, when the massive modes are supposed to decouple, including the complex ones. This question has been posed by one of the present authors in $[18,19]$, but the answer to this question is not known. In the present work we start to explore it by means of the very simple toy model which admits the desired type of decoupling.

The model which we will deal with includes two scalar fields with a strong hierarchy of masses, and was in fact explored by many authors, including at the textbook level [20]. But our purposes require more detailed analysis at the level of effective action, that will be presented below.

The decoupling theorem plays the central role in the effective approach [21]. This theorem [22] states that the contribution of the loop of a field with large mass is quadratically suppressed at low energies. The quadratic decoupling has been explicitly checked in the framework of semiclassical gravity [23, 24], but its generalization to full quantum gravity does not look a simple task. As far as the higher derivative quantum gravity is concerned, the high energy theory has one of many large-mass degrees of freedom which are supposed to decouple in the IR. But the decoupling theorem in its original formulation does not work in this case, because some of the diagrams include internal lines of both light and heavy fields. The questions is what happens with the finite part of such a mixed loop in the IR, when the energy of the fields on the external lines of the diagram is many orders of magnitude below the largest mass in the internal propagators? In principle, as we already mentioned above, the corresponding calculation for the two-scalar model under consideration is known [20], but we shall present it in a slightly different form and also include an external gravitational field.

The paper is organized as follows. In section 2 we formulate the model with two scalars and derive one-loop divergences and $\beta$-functions in the minimal subtraction (MS) scheme of renormalization. As far as the model under consideration is superrenormalizable, these $\beta$-functions are indeed exact, without further contributions at higher loop orders. Starting from the next section, we consider the effective approach, trying to show how the "fundamental" two-scalar theory with cubic interactions matches the effective one-scalar 
model with quartic interaction in the IR. In section 3 we discuss this matching at the tree level. The consideration is performed in curved space and we discuss the subtleties which show up in this case. Section 4 includes derivation of one-loop diagrams with mixed (light and heavy) internal lines. The contents of this section is almost the same as the previous known calculations (see, e.g., [20]), but we add explanations and details which (at least in our opinion) make the result more clear. In section 5 we describe the asymptotic behaviour of the theory in the UV and IR limits. In the former case one meets a perfect correspondence with the MS-scheme results of section 2, and in the IR we can observe how the diagram with mixed internal contents (lines of both heavy and light scalars) boils down to the tadpole diagram of the effective model in the IR. In section 6 the previous results are generalized to the curved space-time, by assuming weak gravitational field and using Riemann normal coordinates, Finally, in section 7 we draw our conclusions.

\section{The model and its MS-scheme renormalization}

In what follows in this section we formulate the classical action, derive one-loop (which are also exact) UV divergences and the full set of $\beta$-functions.

\subsection{Classical action}

Consider the two-scalar model in curved space-time, defined by the following action:

$$
\begin{aligned}
S\left[\chi, \phi^{a}\right]=\int d^{4} x \sqrt{-g}\{ & \frac{1}{2}\left(\nabla \phi^{a}\right)^{2}-\frac{1}{2} m^{2} \phi^{a} \phi^{a}+\frac{1}{2} \xi_{1} R \phi^{a} \phi^{a} \\
& \left.+\frac{1}{2}(\nabla \chi)^{2}-\frac{1}{2} M^{2} \chi^{2}+\frac{1}{2} \xi_{2} R \chi^{2}-\frac{g}{2} \chi \phi^{a} \phi^{a}\right\} .
\end{aligned}
$$

Here $\phi^{a}$ is the $N$-component scalar field $(a=1,2, \ldots, N)$ with a mass $m$, while $\chi$ is a simple real scalar with a mass $M$. Furthermore, $\left(\nabla \phi^{a}\right)^{2}=g^{\mu \nu} \nabla_{\mu} \phi^{a} \nabla_{\nu} \phi^{a},(\nabla \chi)^{2}=g^{\mu \nu} \nabla_{\mu} \chi \nabla_{\nu} \chi$. Furthermore, $\xi_{1,2}$ are nonminimal parameters of interaction between scalars fields and the curvature scalar $R$. Later on we shall see that the quantum arguments require supplementing the action (2.1) with the linear terms (2.7). In this respect the situation is similar to the one for sterile scalar field coupled to fermions [26], but in our case the role of the fermions is played by the second scalar.

\subsection{UV divergences}

At quantum level the action (2.1) leads to the theory with the simple structure of UV divergences. Consider first the minimal subtraction (MS) scheme. The power counting analysis is very simple and it shows that this theory is superrenormalizable, such that the UV divergences can be met only in the first loop and only in the kinetic and massive terms.

We shall use short notations $\int d^{n} x \sqrt{-g} \equiv \int_{x}$, with $n=4$. Let us derive the one-loop divergences. For this end we perform the following shift of the fields into background and quantum counterparts:

$$
\phi^{a} \longrightarrow \phi^{a}+\sigma^{a}, \quad \chi \longrightarrow \chi+\eta .
$$


The one-loop calculation can be done by means of the heat-kernel method, that requires the part of the action bilinear in quantum fields,

$$
\begin{aligned}
& S^{(2)}=\int_{x}\left\{-\frac{1}{2} \sigma^{a} \square \sigma^{a}-\frac{1}{2} \eta \square \eta-\frac{1}{2} m^{2} \sigma^{a} \sigma^{a}-\frac{1}{2} M^{2} \eta^{2}\right. \\
& \left.+\frac{1}{2} \xi_{1} R \sigma^{a} \sigma^{a}+\frac{1}{2} \xi_{2} R \eta^{2}-\frac{g}{2} \chi \sigma^{a} \sigma^{a}-g \eta \sigma^{a} \phi^{a}\right\}
\end{aligned}
$$

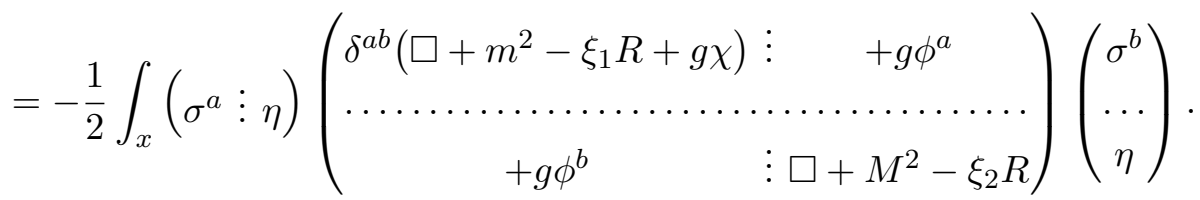

We have to define the matrices

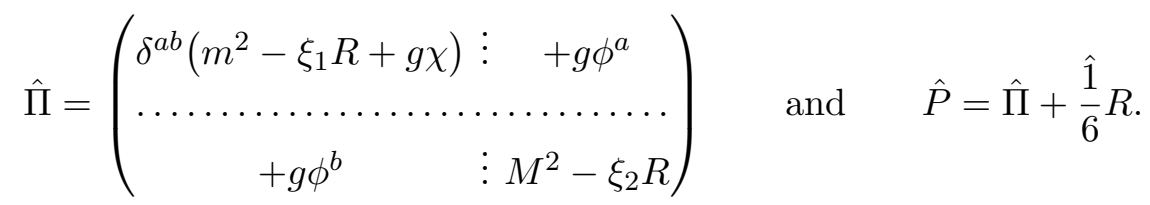

One can note that the vacuum (purely metric-dependent) terms can be easily obtained as sum of the contributions of the two free scalar fields $\varphi^{a}$ and $\chi$, and in general have to interest for us. Neglecting these terms, we meet the general expression for the one-loop divergences

$$
\bar{\Gamma}_{\text {div }}^{(1)}=-\frac{1}{\varepsilon} \mu^{n-4} \int_{x} \operatorname{Tr}\left\{\frac{1}{2} \hat{P}^{2}+\frac{1}{6} \square \hat{P}\right\} .
$$

A small algebra gives us the result

$$
\begin{gathered}
\bar{\Gamma}_{d i v}^{(1)}=-\frac{1}{\epsilon} \mu^{n-4} \int_{x}\left\{g^{2} \phi^{a} \phi^{a}+\frac{N g^{2}}{2} \chi^{2}+N g m^{2} \chi-N g\left(\xi_{1}-\frac{1}{6}\right) \chi R\right. \\
+ \text { total derivative terms }\},
\end{gathered}
$$

where we introduced a compact notation $\epsilon=(4 \pi)^{2}(n-4)$. It is clear that, in order to achieve renormalizable theory, one has to supplement the (2.1) with two more terms,

$$
\Delta S_{l i n}=\int_{x}\left(\alpha \chi+\xi_{3} R \chi\right)
$$

Since these terms are linear in the heavy scalar field, they do not affect the divergences (2.6), defined by the bilinear terms.

\section{$2.3 \beta$-functions}

Using the divergences, one can define the counterterms $\Delta S=-\bar{\Gamma}_{d i v}^{(1)}$, and subsequently the renormalized action,

$$
\begin{aligned}
S_{R}=S+\Delta S & \\
=\int_{x} & \left\{\frac{1}{2}\left(\nabla \phi^{a}\right)^{2}+\frac{1}{2}(\nabla \chi)^{2}+\frac{1}{2} \xi_{1} R \phi^{a} \phi^{a}+\frac{1}{2} \xi_{2} R \chi^{2}-\frac{g}{2} \chi \phi^{a} \phi^{a}-\phi^{a} \phi^{a}\left(\frac{1}{2} m^{2}-\frac{g^{2}}{\epsilon}\right)\right. \\
& \left.\quad-\chi^{2}\left(\frac{1}{2} M^{2}-\frac{N}{2} \frac{g^{2}}{2}\right)+\chi\left(\alpha+\frac{N g m^{2}}{\epsilon}\right)+\chi R\left[\xi_{3}-\frac{N g}{\epsilon}\left(\xi_{1}-\frac{1}{6}\right)\right]\right\}
\end{aligned}
$$


and require it to be equal to the bare action in $n=4$ dimension. This condition boils down to the set of renormalization relations for the fields and one of the masses

$$
\phi_{0}^{a}=\mu^{\frac{n-4}{2}} \phi^{a}, \quad \chi_{0}=\mu^{\frac{n-4}{2}} \chi, \quad m^{2}-\frac{2 g^{2}}{\epsilon}=m_{0}^{2} .
$$

Starting from this point, we can proceed to the derivation of the $\beta$-functions. First of all, we meet the renormalization relation

$$
0=\mu \frac{d m_{0}^{2}}{d \mu}=\mu \frac{d m^{2}}{d \mu}-\frac{2}{\epsilon} \mu \frac{d g^{2}}{d \mu} \Longrightarrow \mu \frac{d m^{2}}{d \mu}=\frac{2}{\epsilon} \mu \frac{d g^{2}}{d \mu} .
$$

On the other hand,

$$
-\frac{g}{2} \mu^{n-4} \chi \phi^{a} \phi^{a}=-\frac{g_{0}}{2} \chi_{0} \phi_{0}^{a} \phi_{0}^{a}
$$

and using (2.9) we get

$$
g_{0}=g \mu^{\frac{4-n}{2}} .
$$

Thus,

$$
\mu \frac{d g_{0}}{d \mu}=0=\mu^{\frac{4-n}{2}} \mu \frac{d g}{d \mu}+\frac{4-n}{2} g \mu^{\frac{4-n}{2}} \Longrightarrow \mu \frac{d g^{2}}{d \mu}=(n-4) g^{2} .
$$

Now, from (2.13) and (2.10) we get

$$
\mu \frac{d m^{2}}{d \mu}=\frac{2 g^{2}}{(4 \pi)^{2}}
$$

Similarly, one can easily derive

$$
\mu \frac{d M^{2}}{d \mu}=\frac{N g^{2}}{(4 \pi)^{2}} .
$$

Furthermore, the renormalization relation

$$
\chi\left(\alpha+\frac{N g m^{2}}{2}\right) \mu^{n-4}=\chi_{0} \alpha_{0}
$$

leads to

$$
0=\mu^{\frac{n-4}{2}}\left[\frac{n-4}{2}\left(\alpha+\frac{N g m^{2}}{\epsilon}\right)+\mu \frac{d \alpha}{d \mu}+\frac{N}{\epsilon} g \mu \frac{d m^{2}}{d \mu}+\frac{N}{\epsilon} \mu \frac{d g}{d \mu} m^{2}\right] .
$$

Since the factor $\frac{d m^{2}}{d \mu}$ in (2.14) is a function of the coupling constant, when substituted into (2.17) it leads to higher order terms of the loop expansion and hence it can be disregarded. Thus,

$$
\mu \frac{d \alpha}{d \mu}=-\frac{n-4}{2} \alpha-\frac{N g m^{2}}{2(4 \pi)^{2}}-\frac{N m^{2}}{\epsilon} \frac{n-4}{2} g \Longrightarrow \beta_{\alpha}=-\frac{N g m^{2}}{(4 \pi)^{2}},
$$

where the limit $n \rightarrow 4$ was taken. In a similar was we obtain, in the same limit,

$$
\beta_{\xi_{3}}=\frac{N g}{(4 \pi)^{2}}\left(\xi_{1}-\frac{1}{6}\right)
$$


for the new nonminimal parameter introduced in (2.7). Let us stress that all other $\beta$ functions vanish and those we derived are exact in the fundamental model (2.1).

Thinking about the correspondence between the $\beta$-functions and non-local form factors in the finite part of effective action, it is clear that such form factors are possible only for the massive terms $m^{2}$ and $M^{2}$, but not for the linear terms with $\alpha$ and $\xi_{3}$. The corresponding $\beta$-functions are, therefore, purely $\overline{M S}$-based, except if we consider non-local surface terms (see $[27,28]$ for an example of the corresponding calculations).

\section{Matching UV and IR at the tree level}

Our main interest is to explore in detail the decoupling in the mixed loops. However it is worthwhile to comment on the consistency of the toy model under consideration.

In the theory (2.1), the potential of the scalar fields in not bounded from below. This represents a critical drawback at the tree level, however there are two possibilities to resolve this issue. First of all, this theory can be also a low-energy sector of an unknown more general model, where the potential becomes healthy and/or the two scalar fields can be composites from some fundamental fermions, for example. On the other hand, one can expect that even treating the model as fundamental, the quantum corrections change the shape of the scalar potential and the effective potential of the theory has well defined vacuum state. Let us start by exploring how this problem is resolved in the IR.

We assume the hierarchy $m \ll M$, such that $\chi$ is a heavy field while $\phi^{a}$ is a set of light fields with equal masses. The idea is to work out the theory at the IR energy scale of the order $m$ and establish an effective IR theory of this, when only the light fields $\phi^{a}$ are propagating. When the energy scale is much below $M$, the oscillations of $\chi$ are suppressed, and one can expect the low-energy action of the form

$$
\Gamma_{\text {eff }}\left[\phi^{a}, g_{\mu \nu}\right]=S\left[\bar{\chi}, \phi^{a}, g_{\mu \nu}\right],
$$

where $\bar{\chi}$ is a particular configuration of the heavy field.

One can also assume that the loops of $\chi$ are small corrections, this point will be further discussed below. Thus, we can disregard the term with cubic self-interaction of this field in the Lagrangian (2.1). Then the on-shell condition can be considered at the tree level in the form

$$
\left.\frac{\delta S\left[\chi, \phi^{a}, g_{\mu \nu}\right]}{\delta \chi}\right|_{\text {classical }}=\left(\square+M^{2}-\xi_{2} R\right) \chi+\frac{g}{2} \phi^{a} \phi^{a}=0 .
$$

Thus, in the classical configuration we have, as an approximation,

$$
\chi=-\frac{g / 2}{\square+M^{2}-\xi_{2} R} \phi^{a} \phi^{a} .
$$

Replacing this solution into the action (2.1) one gets the effective low-energy action of the light field

$$
\begin{aligned}
S_{\text {eff }}\left[\phi^{a}, g_{\mu \nu}\right]=\int d^{4} x \sqrt{-g}\{ & \frac{1}{2}\left(\nabla \phi^{a}\right)^{2}-\frac{1}{2} m^{2} \phi^{a} \phi^{a} \\
& \left.+\frac{1}{2} \xi_{1} R \phi^{a} \phi^{a}+\frac{g^{2}}{8} \phi^{a} \phi^{a} \frac{1}{\square+M^{2}-\xi_{2} R} \phi^{b} \phi^{b}\right\} .
\end{aligned}
$$




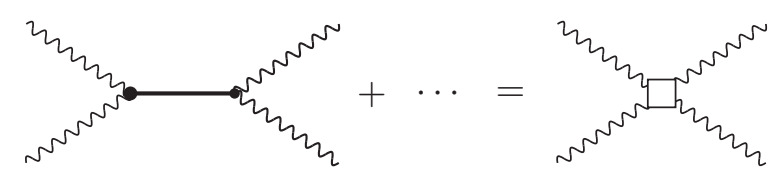

Figure 1. Matching in terms of Feynman diagrams at the tree level. On the left side is the diagram of the fundamental theory, dots referring to different permutations of the momenta. At the right, the diagram corresponds to the effective theory.

The action (3.4) is non-local and resembles the one we meet in the vacuum sector in the consideration of SSB in curved space-time [29]. At the same time this expression becomes local if we make further physical assumptions. ${ }^{1}$

At the energy scale which is much lower than the mass $M$ we can assume that the this mass dominates over the derivatives of the scalar, $\left|\nabla \phi^{a}\right| \ll\left|M \phi^{a}\right|$ and also over the curvature $M^{2} \gg|R|$. In this case one can expand the Green function in a power series

$$
\frac{1}{\square+M^{2}-\xi_{2} R}=\frac{1}{M^{2}}\left[1-\frac{\square-\xi_{2} R}{M^{2}}+\frac{\left(\square-\xi_{2} R\right)^{2}}{M^{4}}+\mathcal{O}\left(\frac{1}{M^{6}}\right)\right],
$$

thus up to the order $1 / M^{6}$ the action of the effective theory of low energies is given by

$$
\begin{aligned}
S_{\mathrm{eff}}\left[\phi^{a}, g_{\mu \nu}\right]=\int d^{4} x \sqrt{-g} & \left\{\frac{1}{2}\left(\nabla \phi^{a}\right)^{2}-\frac{1}{2} m^{2} \phi^{a} \phi^{a}+\frac{1}{2} \xi_{1} R \phi^{a} \phi^{a}\right. \\
& \left.+\frac{g^{2}}{8 M^{2}} \phi^{a} \phi^{a}\left[1-\frac{\square-\xi_{2} R}{M^{2}}+\frac{\left(\square-\xi_{2} R\right)^{2}}{M^{4}}+\ldots\right] \phi^{b} \phi^{b}\right\} .
\end{aligned}
$$

In the leading order this action boils down to the standard action of the light scalar with quartic self-interaction, the effective tree-level Lagrangian being

$$
\mathcal{L}_{\text {eff }}\left[\phi^{a}, g_{\mu \nu}\right]=\frac{1}{2}\left(\nabla \phi^{a}\right)^{2}-\frac{1}{2} m^{2} \phi^{a} \phi^{a}+\frac{1}{2} \xi_{1} R \phi^{a} \phi^{a}-\frac{\lambda}{4 !}\left(\phi^{a} \phi^{a}\right)^{2},
$$

and the tree-level matching of the coupling is

$$
\lambda=-\frac{3 g^{2}}{M^{2}} .
$$

In terms of the Feynman diagrams the matching condition means that the propagation of the heavy field is replaced at low energies by a point interaction as shown in figure 1 . This should already be expected from the expansion of the propagator of field $\chi$ in a series of local operators in (3.5).

Let us note the main difference with the IR matching in the Standard Model of particle physics, where the diagrams with intermediate $W$ and $Z$ bosons, in the IR, become fourfermion interactions in the framework of the Fermi model. In the next after the leading order-approximation in the case of (3.6) we meet an additional curvature-dependent terms, which are not present in the Fermi model of weak interactions. This difference shows that for the scalars in curved space-time one has to introduce an extra condition $M^{2} \gg|R|$, in order to arrive at the effective theory in the IR.

\footnotetext{
${ }^{1}$ Qualitatively similar discussion of the same subject has been given recently in [25].
} 


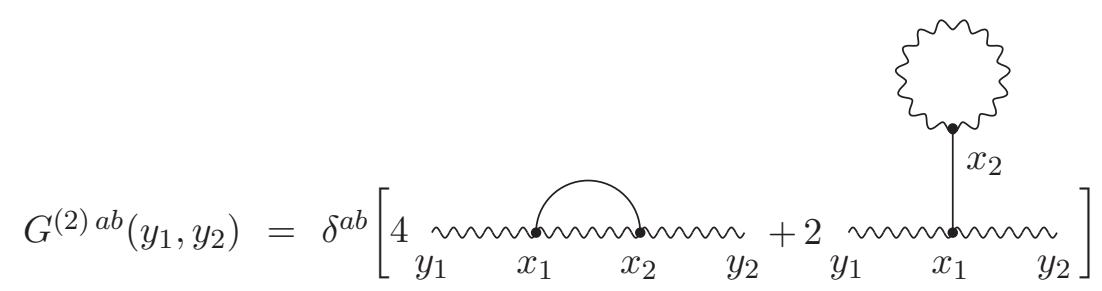

Figure 2. Relevant one-loop diagrams for the two-point function of the field $\phi^{a}$ in the order $g^{2}$ within the fundamental theory in the coordinate space.

\section{One-loop calculations and effective approach}

Let us explore the correspondence between the fundamental theory (2.1) and its effective IR remnant (3.7) at the one-loop level. For the first, simpler calculation, let us start by analysing the problem in the flat space-time, then the Lagrangian of the complete theory is

$$
\mathcal{L}\left[\chi, \phi^{a}\right]=\frac{1}{2}\left(\partial \phi^{a}\right)^{2}-\frac{1}{2} \phi^{a} \phi^{a}+\frac{1}{2}(\partial \chi)^{2}-\frac{1}{2} M^{2} \chi^{2}-\frac{g}{2} \chi \phi^{a} \phi^{a} .
$$

At the tree level the matching condition (3.8).Our first purpose is to generalize this condition to the one-loop approximation.

\subsection{One-loop corrections in full theory}

In order to analyse the IR decoupling of massive degrees of freedom in the theory (4.1), it is useful to consider the diagrams that produce UV divergences and hence are responsible for the $M S$-scheme $\beta$-functions. Thus, in the fundamental theory the corrections of interest are those for the two-point function, that are of the second order in the coupling constant $g$, as shown in figure 2 .

These diagrams have the following analytic representation:

$$
\begin{aligned}
& G^{(2) a b}\left(y_{1}, y_{2}\right)= \\
& =\left(\frac{-i g}{2}\right)^{2} \delta^{a b} \int d^{4} x_{1} d^{4} x_{2}\left[4 i \Delta_{F}^{N}\left(y_{2}-x_{2}\right) i \Delta_{F}^{N}\left(x_{2}-x_{1}\right) i \Delta_{F}\left(x_{2}-x_{1}\right) i \Delta_{F}^{N}\left(x_{1}-y_{1}\right)\right. \\
& \left.\quad+2 i \Delta_{F}^{N}\left(y_{2}-x_{1}\right) i \Delta_{F}^{N}\left(x_{2}-x_{2}\right) i \Delta_{F}\left(x_{2}-x_{1}\right) i \Delta_{F}^{N}\left(x_{1}-y_{1}\right)\right],
\end{aligned}
$$

where

$$
\Delta_{F}\left(x_{1}-x_{2}\right)=\int \frac{d^{4} p}{(2 \pi)^{4}} \frac{e^{-i p\left(x_{1}-x_{2}\right)}}{p^{2}-M^{2}+i \epsilon}
$$

and

$$
\Delta_{F}^{N}\left(x_{1}-x_{2}\right)=\int \frac{d^{4} p}{(2 \pi)^{4}} \frac{e^{-i p\left(x_{1}-x_{2}\right)}}{p^{2}-m^{2}+i \epsilon},
$$

define Feynman propagators for the $\chi$ and $\phi^{a}$ fields, respectively. 


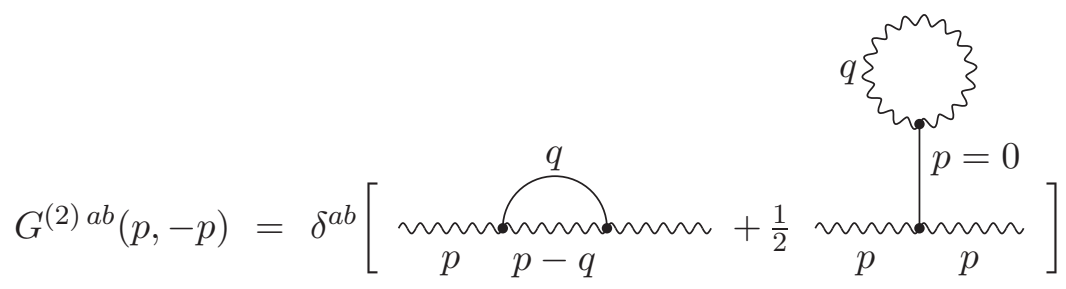

Figure 3. The diagrams for the two-point function in the momentum space.

In the momentum space the expression (4.2) becomes

$$
\begin{aligned}
& G^{(2) a b}(p,-p)= \\
& =\frac{i \delta^{a b}}{\left(p^{2}-m^{2}+i \epsilon\right)}\left\{(-i g)^{2} \int \frac{d^{4} q}{(2 \pi)^{4}} \frac{i}{\left[(p-q)^{2}-m^{2}+i \epsilon\right]} \frac{i}{\left(q^{2}-M^{2}+i \epsilon\right)}\right. \\
& \left.\quad+\frac{(-i g)^{2}}{2} \frac{i}{\left(-M^{2}+i \epsilon\right)} \int \frac{d^{4} q}{(2 \pi)^{4}} \frac{i}{\left(q^{2}-m^{2}+i \epsilon\right)}\right\} \frac{i}{\left(p^{2}-m^{2}+i \epsilon\right)} .
\end{aligned}
$$

The graphical representation in the momentum space is shown in figure 3 .

It proves useful to define, from (4.5),

$$
\Sigma^{1}=(-i g)^{2} \int \frac{d^{4} q}{(2 \pi)^{4}} \frac{i}{\left[(p-q)^{2}-m^{2}+i \epsilon\right]} \frac{i}{\left(q^{2}-M^{2}+i \epsilon\right)}
$$

and

$$
\Sigma^{2}=\frac{(-i g)^{2}}{2} \frac{i}{\left(-M^{2}+i \epsilon\right)} \int \frac{d^{4} q}{(2 \pi)^{4}} \frac{i}{\left(q^{2}-m^{2}+i \epsilon\right)}
$$

so that the correction for the 2-point function is given by the expression

$$
G^{(2) a b}(p,-p)=\frac{i \delta^{a b}}{\left(p^{2}-m^{2}+i \epsilon\right)}\left(\Sigma^{1}+\Sigma^{2}\right) \frac{i}{\left(p^{2}-m^{2}+i \epsilon\right)} .
$$

It is easy to see that the first of these quantities has logarithmic divergence and depends on the external momentum in an essential way. Our purpose is to verify how this expression interpolates between UV and IR regions and what remains from its finite part in the limit $M \rightarrow \infty$. On the other hand, the second (tadpole) diagram is quadratically divergent, but the dependence on the external momentum is trivial.

\subsection{Dimensional regularization}

Using dimensional regularization (see [30] for the introduction), we generalize the divergent expressions in eq. (4.5) to the integrals in $2 \omega$-dimensional Euclidean space,

$$
\Sigma_{2 \omega}^{1}=i g^{2}\left(\mu^{2}\right)^{2-\omega} \int \frac{d^{2 \omega} q}{(2 \pi)^{2 \omega}} \frac{1}{\left[(q-p)^{2}+m^{2}\right]\left(q^{2}+M^{2}\right)},
$$

where $\mu$ is a dimensional renormalization parameter. 
In order to solve $\Sigma_{2 \omega}^{1}$, we use the presentation

$$
\frac{1}{a b}=\int_{0}^{1} \frac{d z}{[a z+b(1-z)]^{2}}
$$

and perform a change of variablel $q^{\prime}=q-p(1-z)$, with $d q^{\prime}=d q$. Thus, $\Sigma_{2 \omega}^{1}$ becomes

$$
\Sigma_{2 \omega}^{1}=i g^{2}\left(\mu^{2}\right)^{2-\omega} \int \frac{d^{2 \omega} q}{(2 \pi)^{2 \omega}} \int_{0}^{1} \frac{d z}{\left[q^{2}+p^{2} z(1-z)+\left(M^{2}-m^{2}\right) z+m^{2}\right]^{2}},
$$

where $q^{\prime}$ was replaced by $q$. The integral over $q$ can be easily taken in a standard way using spherical coordinates in the $2 \omega$-dimensional momentum space. The result is

$$
\Sigma_{2 \omega}^{1}=\frac{i g^{2}}{(4 \pi)^{2}} \Gamma(2-\omega) \int_{0}^{1} d z\left[\frac{4 \pi \mu^{2}}{\left(p^{2} z(1-z)+\left(M^{2}-m^{2}\right) z+m^{2}\right)}\right]^{2-\omega}
$$

where $\Gamma(t)$ is Euler's Gamma function.

Taking the limit $\omega \rightarrow 2$ one can use the expansions

$$
\Gamma(t)=\frac{1}{t}-\gamma+\mathcal{O}(t) \quad \text { and } \quad x^{t}=e^{t \ln x} \simeq 1+t \ln x,
$$

where $\gamma \approx 0,577$ is the Euler-Mascheroni constant. Thus, we arrive at the following result

$$
\Sigma^{1}=\frac{i g^{2}}{(4 \pi)^{2}}\left\{\frac{1}{(2-\omega)}-\gamma-\int_{0}^{1} d z \ln \left[\frac{p^{2} z(1-z)+\left(M^{2}-m^{2}\right) z+m^{2}}{4 \pi \mu^{2}}\right]\right\} .
$$

The integral in the last expression can be easily solved in the form

$$
\int_{0}^{1} d x \ln \left[1+\frac{4 x(1-x)}{a}+4 b x\right]=-2+\frac{(1-a b)}{2} \log (1+4 b)+\frac{A}{2} \log \left[\frac{(A+1)^{2}-a^{2} b^{2}}{(A-1)^{2}-a^{2} b^{2}}\right]
$$

with the restrictions and notation

$$
a>0, \quad b>-1 / 4 \text { and } A=\sqrt{(1+a b)^{2}+a} .
$$

In order to use the result (4.14), we set

$$
a=\frac{4 m^{2}}{p^{2}} \quad \text { and } \quad b=\frac{M^{2}-m^{2}}{4 m^{2}} .
$$

Finally, the first part of the one-loop contribution to the 2-point function is

$$
\begin{gathered}
\Sigma^{1}=\frac{i g^{2}}{(4 \pi)^{2}}\left\{\frac{1}{\varepsilon}+2+\log \left(\frac{\mu^{2}}{m^{2}}\right)-\frac{1-a b}{2} \log \left(\frac{M^{2}}{m^{2}}\right)\right. \\
\left.-\frac{A}{2} \log \left[\frac{(A+1)^{2}-a^{2} b^{2}}{(A-1)^{2}-a^{2} b^{2}}\right]\right\},
\end{gathered}
$$


In the limit $\omega \rightarrow 2$ the result for $\Sigma^{1}$ has divergent and finite parts. It is easy to check that the divergent part corresponds to the result (2.6) in the $\phi^{a} \phi^{a}$ sector. At the same time, the dependence on the external momenta in the finite part is rather complicated and indicates a non-locality of the effective action.

Working out the second integral in (4.5) gives, in the same limit,

$$
\begin{aligned}
\Sigma^{2} & =\frac{i g^{2}}{2} \frac{\left(\mu^{2}\right)^{2-\omega}}{M^{2}} \int \frac{d^{2 \omega} q}{(2 \pi)^{2 \omega}} \frac{1}{q^{2}+m^{2}}=\frac{i g^{2} m^{2}}{2(4 \pi)^{2} M^{2}}\left(\frac{4 \pi \mu^{2}}{m^{2}}\right)^{2-\omega} \Gamma(1-\omega) . \\
& =-\frac{i g^{2}}{2(4 \pi)^{2}} \frac{m^{2}}{M^{2}}\left[\frac{1}{\varepsilon}+1+\log \left(\frac{\mu^{2}}{m^{2}}\right)\right]
\end{aligned}
$$

disregarding $\mathcal{O}(2-\omega)$ terms. There is only a local contribution, as it has to be for the tadpole.

\section{Asymptotic behavior}

Now we are in a position to explore both high-energy and low-energy regimes in the twopoint function. Let's take the UV limit $\left(p^{2} \rightarrow \infty\right)$ in the expression (4.16). The relations $p^{2} \gg m^{2}$ and $p^{2} \gg M^{2}$ result in

$$
\begin{aligned}
& \Sigma_{\mathrm{UV}}^{1}\left(p^{2} \rightarrow \infty\right) \\
& =\frac{i g^{2}}{(4 \pi)^{2}}\left\{\frac{1}{\varepsilon}+2-\log \left(\frac{p^{2}}{\mu^{2}}\right)-\frac{m^{2}}{p^{2}}\left[1+\log \left(\frac{p^{2}}{m^{2}}\right)\right]-\frac{M^{2}}{p^{2}}\left[1+\log \left(\frac{p^{2}}{M^{2}}\right)\right]\right\},
\end{aligned}
$$

where all lower order terms are omitted. The logarithmic terms in the form factor are proportional to the divergence, as it should be in the UV. Furthermore, it is easy to check that the divergent term exactly correspond the result in eq. (2.6). This correspondence has a relevant consequence. Let us remember that the theory is superrenormalizable and that eq. (2.6) give all UV divergences which we can meet in all loop orders. This means that higher loop corrections to (5.1) are also finite and, moreover, they do not have higher order logarithmic corrections. Thus, eq. (5.1) is the leading contribution not only at the one-loop level, but also non-perturbatively.

In the IR we assume $p^{2} \ll M^{2}$ in the expression (4.16). In the leading order in $p^{2}$ this yields

$$
\Sigma_{\mathrm{IR}}^{1}\left(M^{2} \gg p^{2}\right)=\frac{i g^{2}}{(4 \pi)^{2}}\left\{\frac{1}{\varepsilon}+1+\log \left(\frac{\mu^{2}}{M^{2}}\right)+\frac{m^{2}}{M^{2}} \log \left(\frac{m^{2}}{M^{2}}\right)-\frac{1}{2} \frac{p^{2}}{M^{2}}\right\} .
$$

It is easy to see that there is no nonlocal part with a logarithmic form factor. Thus, the diagram with mixed (light and heavy fields) internal lines boils down to the tadpole-type contribution in the IR.

\subsection{Matching with IR at the one-loop level}

Once we know the one-loop behavior of the fundamental theory in the IR, it is possible to establish the correspondence between this result and that obtained from an effective theory, 


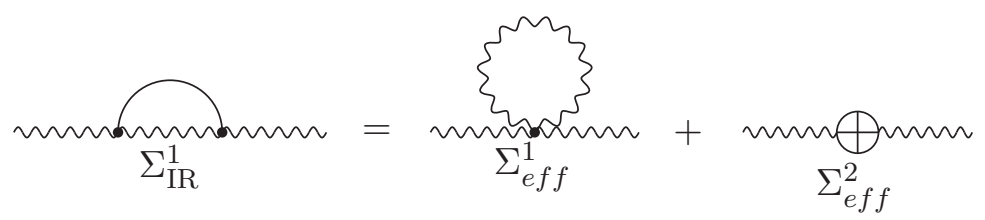

Figure 4. Illustration of the one-loop match in the IR between fundamental theory (left side of equality) and the effective theory with the four-scalar interaction (right side of equality).

taking into account only the quartic interaction of the field $\phi^{a}$ at the one-loop level. At the first stage, we shall disregard the correction (4.18) for the fundamental theory, since this contribution is independent of the momentum. The graphical representation of the one-loop matching is shown in figure 4.

On the l.h.s., $\Sigma_{\mathrm{IR}}^{1}$ is the correction to the propagator of the $\phi^{a}$ field in the fundamental theory in the IR limit (5.2). On the r.h.s., $\Sigma_{\text {eff }}^{1}$ is the one-loop correction for the propagator in the effective theory, whereas $\Sigma_{\text {eff }}^{2}$ is an additional term (established below) representing the difference between the one-loop correction of the fundamental theory in the low energies and the correction to one-loop of the effective theory of low energies.

The effective theory in the IR, at the tree level corresponds to a quartic interaction of $N$ scalars fields (3.7) in the flat space,

$$
\mathcal{L}_{\mathrm{eff}}\left[\phi^{a}\right]=\frac{1}{2}\left(\partial \phi^{a}\right)^{2}-\frac{1}{2} m^{2} \phi^{a} \phi^{a}-\frac{\lambda}{4 !}\left(\phi^{a} \phi^{a}\right)^{2},
$$

where $\lambda=-\frac{3 g^{2}}{M^{2}}$. The one-loop contribution in this effective theory can be easily calculated to give

$$
\Sigma_{\text {eff }}^{1}=-\frac{3 i g^{2} m^{2}}{2(4 \pi)^{2} M^{2}}\left[\frac{1}{\varepsilon}+1+\log \left(\frac{\mu^{2}}{m^{2}}\right)\right]
$$

with $\frac{1}{\varepsilon}$ being defined in (4.17).

The additional term $\Sigma_{\text {eff }}^{2}$ is obtained by inserting new coefficients in the effective Lagrangian

$$
\mathcal{L}_{\text {eff }}\left[\phi^{a}\right]=\frac{1}{2}\left(1+C_{\phi}\right)\left(\partial \phi^{a}\right)^{2}-\frac{1}{2}\left(m^{2}+C_{m^{2}}\right) \phi^{a} \phi^{a}-\frac{\lambda}{4 !}\left(\phi^{a} \phi^{a}\right)^{2},
$$

providing the vertices

$$
\Sigma_{\text {eff }}^{2}=-i C_{m^{2}}+i p^{2} C_{\phi}
$$

These coefficients should not be confused with the counterterms. Their role is not to remove divergences from theory, but to ensure that at low energies both effective and fundamental theory lead to identical results, as shown below.

The divergences in (5.2) and (5.4) can be removed by a suitable renormalization. Since the renormalization scale is arbitrary, we are interested only in the finite part of these corrections. The one-loop matching is achieved by

$$
\Sigma_{\mathrm{IR}}^{1}=\Sigma_{\mathrm{eff}}^{1}+\Sigma_{\mathrm{eff}}^{2}
$$

remembering that we are interested in the low energy regime of the fundamental theory. 
The equality (5.7) leads to the following values,

$$
C_{m^{2}}=-\frac{g^{2}}{(4 \pi)^{2}}\left[\left(1+\frac{m^{2}}{M^{2}}\right)\left(1+\log \frac{\mu^{2}}{M^{2}}\right)+\frac{m^{2}}{2 M^{2}}\left(1+\log \frac{\mu^{2}}{m^{2}}\right)\right]
$$

and

$$
C_{\phi}=-\frac{g^{2}}{2(4 \pi)^{2} M^{2}}
$$

These results for $C_{m^{2}}$ and $C_{\phi}$ show how the effective theory differs from the fundamental theory of low energies. It is important to note that these two terms are momentumindependent, and therefore can be compensated by the change in renormalization condition.

It is easy to see from the expression of the tadpole (4.18), that if $\Sigma^{2}$ is added on the left side of (5.7) the last term of $C_{m^{2}}$ cancels and we arrive at

$$
C_{m^{2}}=-\frac{g^{2}}{(4 \pi)^{2}}\left(1+\frac{m^{2}}{M^{2}}\right)\left(1+\log \frac{\mu^{2}}{M^{2}}\right) .
$$

When $M^{2} \rightarrow \infty$ the results for $C_{m^{2}}$ and $C_{\phi}$ confirm the decoupling theorem. In the IR, the difference between fundamental and effective theories is reduced by renormalization of UV divergences by irrelevant local counterterms, and in the terms proportional to the inverse of the square of the mass of the heavy field.

From (5.5) and the results obtained by the IR matching is possible to note that the effective theory can be written as

$$
\mathcal{L}_{\text {eff }}=\frac{1}{2} C_{1}\left(\partial \phi^{a}\right)^{2}-\frac{1}{2} C_{2} \phi^{a} \phi^{a}-\frac{1}{4 !} C_{3}\left(\phi^{a} \phi^{a}\right)^{2},
$$

where the coefficients $C_{1}, C_{2}$ and $C_{3}$ depend on the parameters of the fundamental theory valid in any energy scale. In general, these coefficients can be constructed order by order in the loop expansion, ensuring that the results of the two theories are equivalent at low energies.

\section{Decoupling in a weak gravitational field}

In this section we will generalize the previous considerations to the same theory in a curved space. As usual, the derivation of non-local form factors requires that the metric corresponds to the almost flat geometry. Then, the external metric can be treated as a small perturbation. Alternatively, one can make an expansion of the 2-point function in normal coordinates [31] and directly arrive at the formally covariant result for the form factor. In this section we shall follow this approach and use the expansion in the linear order in curvature tensor components.

Starting from the first term of (4.2), we write the 2-point function in the form

$$
\begin{aligned}
G^{(2) a b}\left(y_{1}, y_{2}\right)= & \delta^{a b} \int d^{4} x_{2} \sqrt{-g\left(x_{2}\right)} \int d^{4} x_{1} \sqrt{-g\left(x_{1}\right)} \\
& \times G^{N}\left(y_{2}, x_{2}\right)\left[(-i g)^{2} G^{N}\left(x_{2}, x_{1}\right) G\left(x_{2}, x_{1}\right)\right] G^{N}\left(x_{1}, y_{1}\right)
\end{aligned}
$$


where $G^{N}(x, y)=i \Delta_{F}^{N}(x-y)$ and $G(x, y)=i \Delta_{F}(x-y)$ are the flat-space Green functions for the light and heavy fields, respectively.

After Wick rotation to Euclidean space, this 2-point function can be written as

$$
G^{(2) a b}\left(y_{1}, y_{2}\right)=-i \delta^{a b} \int_{x_{2}} \int_{x_{1}} G^{N}\left(y_{2}, x_{2}\right) \Sigma^{1, R} G^{N}\left(x_{1}, y_{1}\right)
$$

where

$$
\Sigma^{1, R}=i g^{2} G^{N}\left(x_{2}, x_{1}\right) G\left(x_{2}, x_{1}\right) .
$$

Let us note that $\Sigma^{1, R}$ includes the product of the two propagators that appear in the loop. These propagators can be expanded in terms of normal coordinates in a curved space according to the well-known result of [31], which in $2 \omega$-dimensions provides

$$
\Sigma^{1, R}=i g^{2} \int \frac{d^{2 \omega} q}{(2 \pi)^{2 \omega}} e^{i q\left(x_{2}-x_{1}\right)} G(q, m) \int \frac{d^{2 \omega} p}{(2 \pi)^{2 \omega}} e^{i p\left(x_{2}-x_{1}\right)} G(p, M),
$$

where $\Sigma^{1, R}=\Sigma^{1, R}(k), q+p=k$,

$$
G(q)=\frac{1}{q^{2}+m^{2}}+\frac{\tilde{\xi}_{1} R}{\left(q^{2}+m^{2}\right)^{2}}-\frac{2}{3} \frac{R_{\alpha \beta} q^{\alpha} q^{\beta}}{\left(q^{2}+m^{2}\right)^{3}}+\mathcal{O}\left(q^{-5}\right)
$$

is the standard expansion of the propagator [31], and we are using notations

$$
\tilde{\xi}_{1}=\frac{1}{3}-\xi_{1} \quad \text { and } \quad \tilde{\xi}_{2}=\frac{1}{3}-\xi_{2}
$$

Let us note that the expansion of the vertices in normal coordinates in eq. (6.4) is not necessary, as explained below.

Consider the dimensional regularization of the integrals in eq. (6.4), using the transformations for the integrals with vector symmetry described in the books [20,32]. Our final purpose is to evaluate these integrals in the IR limit with $m^{2} \ll M^{2}$ and $p^{2} \ll M^{2}$. Setting $x_{2}-x_{1}=y$, after some algebra we obtain

$$
\begin{aligned}
I_{1} & =\int \frac{d^{2 \omega} k}{(2 \pi)^{2 \omega}} e^{i k y} \int \frac{d^{2 \omega} p}{(2 \pi)^{2 \omega}} \frac{1}{\left[(p-k)^{2}+m^{2}\right]\left(p^{2}+M^{2}\right)} \\
& =\frac{1}{(4 \pi)^{2}} \int \frac{d^{4} k}{(2 \pi)^{4}} e^{i k y}\left[\frac{1}{\varepsilon}+1+\log \left(\frac{\mu^{2}}{M^{2}}\right)+\frac{m^{2}}{M^{2}} \log \left(\frac{m^{2}}{M^{2}}\right)-\frac{1}{2} \frac{k^{2}}{M^{2}}\right] .
\end{aligned}
$$

This is the same expression as (5.2), that means for $R=0$ we recover the flat space result. 
Other integrals correspond to the dependence on the curvature,

$$
\begin{aligned}
I_{2} & =\tilde{\xi}_{1} R \int \frac{d^{2 \omega} k}{(2 \pi)^{2 \omega}} e^{i k y} \int \frac{d^{2 \omega} q}{(2 \pi)^{2 \omega}} \frac{1}{\left[(q-k)^{2}+M^{2}\right]\left(q^{2}+m^{2}\right)^{2}} \\
& =\frac{\tilde{\xi}_{1} R}{(4 \pi)^{2}} \int \frac{d^{4} k}{(2 \pi)^{4}} \frac{e^{i k y}}{M^{2}}\left[-1-\log \left(\frac{m^{2}}{M^{2}}\right)\right] \\
I_{3} & =-\frac{2}{3} R^{\alpha \beta} \int \frac{d^{2 \omega} k}{(2 \pi)^{2 \omega}} e^{i k y} \int \frac{d^{2 \omega} q}{(2 \pi)^{2 \omega}} \frac{q_{\alpha} q \beta}{\left(q^{2}+m^{2}\right)^{3}\left[(q-k)^{2}+M^{2}\right]} \\
& =-\frac{1}{6} \frac{R}{(4 \pi)^{2}} \int \frac{d^{4} k}{(2 \pi)^{4}} \frac{e^{i k y}}{M^{2}}\left[-\frac{3}{2}-\log \left(\frac{m^{2}}{M^{2}}\right)\right] \\
I_{4} & =\tilde{\xi_{2}} R \int \frac{d^{2 \omega} k}{(2 \pi)^{2 \omega}} e^{i k y} \int \frac{d^{2 \omega} q}{(2 \pi)^{2 \omega}} \frac{1}{\left(q^{2}+m^{2}\right)\left[(q-k)^{2}+M^{2}\right]^{2}} \\
& =\frac{\tilde{\xi_{2}} R}{(4 \pi)^{2}} \int \frac{d^{4} k}{(2 \pi)^{4}} e^{i k y} \frac{1}{M^{2}} \\
I_{5} & =-\frac{2}{3} R^{\alpha \beta} \int \frac{d^{2 \omega} k}{(2 \pi)^{2 \omega}} e^{i k y} \int \frac{d^{2 \omega} q}{(2 \pi)^{2 \omega}} \frac{\left(k_{\alpha}-q_{\alpha}\right)\left(k_{\beta}-q_{\beta}\right)}{\left(q^{2}+m^{2}\right)\left[(q-k)^{2}+M^{2}\right]^{3}} \\
& =-\frac{1}{6} \frac{R}{(4 \pi)^{2}} \int \frac{d^{4} k}{(2 \pi)^{4}} e^{i k y} \frac{1}{2 M^{2}},
\end{aligned}
$$

where we have set $\omega=2$, since there are no divergences.

Disregarding the $k^{2}$-term in eq. (6.7), the combination of these integrals gives

$$
\begin{aligned}
\Sigma_{\mathrm{IR}}^{1, R}=\delta(y) \frac{i g^{2}}{(4 \pi)^{2}}\{ & \frac{1}{\varepsilon}+\log \left(\frac{1}{M^{2}}\right)+\frac{m^{2}}{M^{2}} \log \left(\frac{m^{2}}{M^{2}}\right) \\
& \left.+\frac{R}{M^{2}}\left[\left(\xi_{1}-\frac{1}{6}\right) \log \left(\frac{m^{2}}{M^{2}}\right)+\xi_{1}-\xi_{2}+\frac{1}{6}\right]\right\} .
\end{aligned}
$$

An important observation is in order. The expression (6.4) does not include the expansion of the vertex in normal coordinates. The reason is that the expansion of the vertex comes from the factors of $\sqrt{g}$ in the interaction terms. In one of the points (e.g. $x_{1}$ ) the metric is flat, such that $\sqrt{g\left(x_{1}\right)}=1$, and in another point the expansion boils down to the factor of $\sqrt{g}$ in the final expression for $\Sigma_{\mathrm{IR}}^{1, R}$ in eq. (6.9), since this is a local expression that has an extra factor of $\delta_{c}\left(x_{2}-x_{1}\right)$. This one should be a covariant delta function in normal coordinates, absorbing the whole factor of $\sqrt{g}$, that comes from the second vertex. The delta function deletes one of the integrals in eq. (6.2), such that the results becomes a local expression.

In the IR limit, the 2-point function in (6.2) can be written in the form

$$
G^{(2) a b}\left(y_{1}, y_{2}\right)=-i \delta^{a b} \int d^{4} x \sqrt{g(x)} G^{N}\left(y_{2}, x\right) \Sigma_{\mathrm{IR}}^{1, R} G^{N}\left(x, y_{1}\right),
$$

where $\Sigma_{\mathrm{IR}}^{1, R}$ no longer proportional to the delta function. It is easy to see that the expression (6.10) has UV divergences only in the flat-space sector, while the terms with scalar curvature are finite. This output is in a perfect correspondence with the covariant calculation in section 2 . 
In order to compare the two approaches to the description of the IR, let us now consider an effective theory with quartic interaction in curved space. Such a the theory of the field $\phi^{a}$ leads to the one-loop contribution

$$
G_{\mathrm{eff}}^{(2) a b}\left(y_{1}, y_{2}\right)=-i \delta^{a b} \int d^{4} x \sqrt{g(x)} G^{N}\left(y_{2}, x\right)\left[-\frac{i \lambda}{2} G^{N}(x, x)\right] G^{N}\left(x, y_{1}\right) .
$$

This expression can be directly compared to (6.10), to show that both have the same structure in terms of propagators. This comparison is possible by the fact that in low energies the fundamental theory has only one vertex.

The matching at the one-loop level in the curved space can be done in pretty much the same way as in flat space,

$$
\Sigma_{\mathrm{IR}}^{1, R}=\Sigma_{\mathrm{eff}}-i C_{m^{2}}+i C_{R}
$$

Here $\Sigma_{\text {eff }}$ is defined from (6.11) as

$$
\Sigma_{\text {eff }}=-\frac{i \lambda}{2} G^{N}\left(x^{\prime}, x^{\prime}\right)
$$

Using the expansion in normal coordinates, we get

$$
\Sigma_{\mathrm{eff}}=-\frac{i \lambda}{2} \int \frac{d^{2 \omega} p}{(2 \pi)^{2 \omega}}\left\{\frac{1}{p^{2}+m^{2}}+\frac{\tilde{\xi_{1}} R}{\left(p^{2}+m^{2}\right)^{2}}-\frac{2}{3} \frac{R_{\alpha \beta} p^{\alpha} p^{\beta}}{\left(p^{2}+m^{2}\right)^{3}}+\mathcal{O}\left(p^{-5}\right)\right\}
$$

that leads to the result

$$
\Sigma_{\text {eff }}=\frac{i \lambda}{2(4 \pi)^{2}}\left\{m^{2}\left[\frac{1}{\varepsilon}+1+\log \left(\frac{\mu^{2}}{m^{2}}\right)\right]+\left(\xi_{1}-\frac{1}{6}\right) R\left[\frac{1}{\varepsilon}+\log \left(\frac{\mu^{2}}{m^{2}}\right)\right]\right\} .
$$

Note that, according to eq. (3.8), even in curved space we have $\lambda=-\frac{3 g^{2}}{M^{2}}$. Thus, the flat part of the IR matching condition in (6.12) is satisfied with the $C_{m^{2}}$ from (5.8). On the other hand, there is an additional matching condition in the first order in curvature,

$$
C_{R}=\frac{g^{2}}{(4 \pi)^{2}} \frac{R}{M^{2}}\left[\left(\xi_{1}-\frac{1}{6}\right) \log \left(\frac{\mu^{2}}{M^{2}}\right)+\frac{1}{2}\left(\xi_{1}-\frac{1}{6}\right) \log \left(\frac{\mu^{2}}{m^{2}}\right)+\left(\xi_{1}-\xi_{2}\right)+\frac{1}{6}\right] .
$$

As in the flat space, we can consider the correction of tadpole to the fundamental theory, given by the second term of (4.2) in curved space. In Euclidean space it is

$$
G_{\text {tadpole }}^{(2) a b}\left(y_{1}, y_{2}\right)=-i \delta^{a b} \int_{x_{2}} \int_{x_{1}} G^{N}\left(y_{2}, x_{1}\right)\left[\Sigma^{2, R}\right] G^{N}\left(x_{1}, y_{1}\right),
$$

with

$$
\begin{aligned}
\Sigma^{2, R} & =\frac{i g^{2}}{2} G\left(x_{2}, x_{1}\right) G^{N}\left(x_{2}, x_{2}\right) \\
& =\frac{i g^{2}}{2} \int \frac{d^{4} p}{(2 \pi)^{2}} \frac{e^{i p \cdot\left(x_{2}-x_{1}\right)}}{M^{2}}\left[1+\mathcal{O}\left(\frac{p^{2}}{M^{2}}\right)\right] G^{N}\left(x_{2}, x_{2}\right) \\
& =\frac{i g^{2}}{2 M^{2}} \delta_{c}(y) \int \frac{d^{2 \omega} q}{(2 \pi)^{\omega}}\left[\frac{1}{q^{2}+m^{2}}+\frac{\xi_{1} R}{\left(q^{2}+m^{2}\right)^{2}}-\frac{2}{3} \frac{R_{\alpha \beta} q^{\alpha} q^{\beta}}{\left(q^{2}+m^{2}\right)^{3}}+\mathcal{O}\left(q^{-5}\right)\right] .
\end{aligned}
$$


In this expression, the covariant delta function emerges in the low energy limit, when expanding the propagator of the heavy field in powers of $\frac{p^{2}}{M^{2}}$ and disregard higher orders in the expansion.

The tadpole part gives the contribution

$$
\Sigma^{2, R}=-\frac{g^{2}}{2(4 \pi)^{2}}\left\{\frac{m^{2}}{M^{2}}\left[\frac{1}{\varepsilon}+1+\log \left(\frac{\mu^{2}}{m^{2}}\right)\right]+\left(\xi_{1}-\frac{1}{6}\right) \frac{R}{M^{2}}\left[\frac{1}{\varepsilon}+\log \left(\frac{\mu^{2}}{m^{2}}\right)\right]\right\} .
$$

Adding this result to the l.h.s. of (6.12), the flat part of the matching at one-loop level leads to the expression (5.10), while the curvature-dependent part gives

$$
C_{R}=\frac{g^{2}}{(4 \pi)^{2}} \frac{R}{M^{2}}\left[\left(\xi_{1}-\frac{1}{6}\right) \log \left(\frac{\mu^{2}}{M^{2}}\right)+\left(\xi_{1}-\xi_{2}\right)+\frac{1}{6}\right] .
$$

\section{Conclusions}

Using a very simple model with two scalar fields, we explored the behavior of the diagrams with mixed internal lines. To some extent the results are not new (see, e.g., [20]), but we made the calculations keeping in focus the effective action approach, the relevant problem of decoupling of higher derivatives in quantum gravity [19] and considered in full details the matching between UV and IR, including in the weak external gravitational field.

The main output of our investigation is that the contribution of the self-energy type, one-loop diagram with one internal line of the light field and another one of the field with the large mass, in the far IR boils down to the tadpole contribution, that does not produce a non-local form factor. In the toy model under consideration this means that the self-energy diagram in the "fundamental" model with two types of scalars produce a standard non-local form factor with the logarithmic asymptotic behavior in the UV, but in the IR there is no relevant form factor and the results is essentially the same tadpole-type contribution that one can get in the effective low-energy model with a single-type light scalar field. The same qualitative situation holds in a weak gravitational field. Indeed, due to the superrenormalizable nature of the fundamental model, the logarithmic asymptotic behavior in the UV is not possible for the curvature-dependent terms. However, in the IR we observe a perfect matching between effective and fundamental models, that confirms the main results of our work.

From the gravitational perspective, the massive fields are ghosts and tachyons that are present in the higher derivative versions of quantum gravity. In this respect the important question is whether the IR effective theory is always the quantum general relativity, or it can be some other, e.g., non-local model, as it was discussed in [19]. Making a "continuation" of our present result implies that one can expect that the mixed-content diagrams become irrelevant in the IR. Then the transferred momentum is the unique IR regulator and this means that the quantum general relativity is expected to be a universal model of IR quantum gravity, as it was expected in the paper by Donoghue [33, 34] and many consequent works (see, e.g., the reviews [35, 36]). Indeed, this kind of conclusion should be seen as a well-motivated conjecture, and its verification would be an interesting work to be done. 


\section{Note added}

Regardless we mainly use the theory (2.1) as a toy model for quantum gravity, it is worthy to discuss whether this model can be independently applied to the description of some physical phenomena. The idea looks attractive, because i) it is superrenormalizable and therefore does not need complicated nonperturbative treatment. On the other hand, the standard four-scalar model emerge naturally in the IR, as we have seen in section 2. This situation should enable one to avoid the well-known difficulties with the stability of Higgs potential in the UV (see e.g. [37] and [38]).

Unfortunately, this apparently nice plan meets two serious obstacles. First of all, the classical potential of the scalar fields in the theory (2.1) is not bounded from below and, therefore, its quantum mechanical formulation meets a fundamental difficulty. Moreover, since the theory is superrenormalizable, the loop contributions to the quantum effective potential only enhance logarithmically the massive terms. Thus, even in the non-perturbative effective potential one should expect the directions that make the potential unbounded, that confirm the tree-level verdict. Second, if we assume that the scalar fields $\phi^{a}$ and $\chi$ couple to fermions, the nice features of the model immediately break down, as the renormalizability should require $\left(\phi^{a} \phi^{a}\right)^{2}, \phi^{a} \phi^{a} \chi^{2}$ and $\chi^{4}$ terms to be introduced, and then the theory is not superrenormalizable anymore.

Thus, there is no much chances to transform our model into the base of the fundamental theory behind the Standard Model of Particle Physics (SM). At the same time, the simplified versions of the model (2.1) are known as useful tools in cosmology. E.g., in the well-known papers [39, 40] (see further references therein, also the recent work [41]) the scalar field $\chi$ describes the Bose-Einstein condensate of some more fundamental fields. The considerations in these works involves not only tree-level, but also the loop effects. Thus, it is possible that the consistent formulation of the model in curved space which we presented in sections 2 and 3, may be useful for further developments of this approach, the same concerns its IR quantum behavior, that we discussed in the subsequent sections.

Finally, let us mention the possibility that the large-mass field $\chi$ in our physically motivated toy model may turn out to be a natural concept in the models of composite Higgs. Up to some extent, this can be a particle physics version of the cosmological applications considered in [39]. Once again, in this case it may be useful to have a consistent formulation of the model in curved space, e.g. because it may put an additional restrictions of the heavy scalar field as a composite object coming from some fundamental fermions beyond the SM, for example.

\section{Acknowledgments}

Authors are grateful to Blaženka Melić and Oleg Antipin from RBI/Zagreb for useful discussions and clarifications concerning the effective approach in particle physics. The work of I.Sh. was partially supported by Conselho Nacional de Desenvolvimento Científico e Tecnológico (CNPq) under the grant 303635/2018-5 and by Fundação de Amparo à Pesquisa de Minas Gerais (FAPEMIG) under the project APQ-01205-16. 
Open Access. This article is distributed under the terms of the Creative Commons Attribution License (CC-BY 4.0), which permits any use, distribution and reproduction in any medium, provided the original author(s) and source are credited.

\section{References}

[1] S. Weinberg, Effective Gauge Theories, Phys. Lett. 91B (1980) 51 [InSPIRE].

[2] A.V. Manohar, Effective field theories, Lect. Notes Phys. 479 (1997) 311 [hep-ph/9606222] [INSPIRE].

[3] A. Pich, Effective field theory: Course, in Probing the standard model of particle interactions. Proceedings, Summer School in Theoretical Physics, NATO Advanced Study Institute, 68th session, Les Houches, France, July 28-September 5, 1997. Pt. 1, 2, pp. 949-1049 (1998) [hep-ph/9806303] [INSPIRE].

[4] C.P. Burgess, Quantum gravity in everyday life: General relativity as an effective field theory, Living Rev. Rel. 7 (2004) 5 [gr-qc/0311082] [INSPIRE].

[5] J.Z. Simon, Higher Derivative Lagrangians, Nonlocality, Problems and Solutions, Phys. Rev. D 41 (1990) 3720 [INSPIRE].

[6] L. Parker and J.Z. Simon, Einstein equation with quantum corrections reduced to second order, Phys. Rev. D 47 (1993) 1339 [gr-qc/9211002] [INSPIRE].

[7] F.d.O. Salles and I.L. Shapiro, Do we have unitary and (super)renormalizable quantum gravity below the Planck scale?, Phys. Rev. D 89 (2014) 084054 [Erratum ibid. D 90 (2014) 129903] [arXiv: 1401.4583] [inSPIRE].

[8] A. Accioly, B.L. Giacchini and I.L. Shapiro, Low-energy effects in a higher-derivative gravity model with real and complex massive poles, Phys. Rev. D 96 (2017) 104004 [arXiv: 1610.05260$]$ [INSPIRE].

[9] A. Accioly, B.L. Giacchini and I.L. Shapiro, On the gravitational seesaw in higher-derivative gravity, Eur. Phys. J. C 77 (2017) 540 [arXiv:1604.07348] [INSPIRE].

[10] M. Asorey, J.L. López and I.L. Shapiro, Some remarks on high derivative quantum gravity, Int. J. Mod. Phys. A 12 (1997) 5711 [hep-th/9610006] [inSPIRE].

[11] L. Modesto and I.L. Shapiro, Superrenormalizable quantum gravity with complex ghosts, Phys. Lett. B 755 (2016) 279 [arXiv:1512.07600] [InSPIRE].

[12] L. Modesto, Super-renormalizable or finite Lee-Wick quantum gravity, Nucl. Phys. B 909 (2016) 584 [arXiv: 1602.02421] [INSPIRE].

[13] N.V. Krasnikov, Nonlocal gauge theories, Theor. Math. Phys. 73 (1987) 1184 [InSPIRE].

[14] Y.V. Kuz'min, The convergent nonlocal gravitation (in Russian), Sov. J. Nucl. Phys. 50 (1989) 1011 [INSPIRE].

[15] E.T. Tomboulis, Nonlocal and quasilocal field theories, Phys. Rev. D 92 (2015) 125037 [arXiv: 1507.00981] [INSPIRE].

[16] E.T. Tomboulis, Superrenormalizable gauge and gravitational theories, hep-th/9702146 [INSPIRE].

[17] I.L. Shapiro, Counting ghosts in the "ghost-free" non-local gravity, Phys. Lett. B 744 (2015) 67 [arXiv: 1502.00106] [INSPIRE]. 
[18] G. de Berredo-Peixoto and I.L. Shapiro, Higher derivative quantum gravity with Gauss-Bonnet term, Phys. Rev. D 71 (2005) 064005 [hep-th/0412249] [InSPIRE].

[19] I.L. Shapiro, Polemic Notes On IR Perturbative Quantum Gravity, Int. J. Mod. Phys. A 24 (2009) 1557 [arXiv: 0812.3521] [inSPIRE].

[20] V. Ilisie, Concepts in Quantum Field Theory. A Practitioner's Toolkit, Springer (2016).

[21] S. Hartmann, Effective field theories, reductionism and scientific explanation, Stud. Hist. Phil. Sci. B 32 (2001) 267 [INSPIRE].

[22] T. Appelquist and J. Carazzone, Infrared Singularities and Massive Fields, Phys. Rev. D 11 (1975) 2856 [INSPIRE].

[23] E.V. Gorbar and I.L. Shapiro, Renormalization group and decoupling in curved space, JHEP 02 (2003) 021 [hep-ph/0210388] [INSPIRE].

[24] E.V. Gorbar and I.L. Shapiro, Renormalization group and decoupling in curved space. 2. The Standard model and beyond, JHEP 06 (2003) 004 [hep-ph/0303124] [INSPIRE].

[25] Ł. Nakonieczny, Curved spacetime effective field theory (cEFT) - construction with the heat kernel method, JHEP 01 (2019) 034 [arXiv:1811.01656] [INSPIRE].

[26] V. Fernandes Barra, I.L. Buchbinder, J.G. Joaquim, A.R. Rodrigues and I.L. Shapiro, Renormalization of Yukawa model with sterile scalar in curved spacetime, Eur. Phys. J. C 79 (2019) 458 [arXiv: 1903.11546] [INSPIRE].

[27] S.A. Franchino-Viñas, T. de Paula Netto, I.L. Shapiro and O. Zanusso, Form factors and decoupling of matter fields in four-dimensional gravity, Phys. Lett. B 790 (2019) 229 [arXiv: 1812.00460] [INSPIRE].

[28] S.A. Franchino-Viñas, T. de Paula Netto and O. Zanusso, Vacuum effective actions and mass-dependent renormalization in curved space, Universe 5 (2019) 67 [arXiv:1902.03167] [INSPIRE].

[29] E.V. Gorbar and I.L. Shapiro, Renormalization group and decoupling in curved space. 3. The Case of spontaneous symmetry breaking, JHEP 02 (2004) 060 [hep-ph/0311190] [INSPIRE].

[30] G. Leibbrandt, Introduction to the Technique of Dimensional Regularization, Rev. Mod. Phys. 47 (1975) 849 [INSPIRE].

[31] T.S. Bunch and L. Parker, Feynman Propagator in Curved Space-Time: A Momentum Space Representation, Phys. Rev. D 20 (1979) 2499 [InSPIRE].

[32] M.D. Schwartz, Quantum field theory and the standard model, Cambridge University Press, New York (2014) [INSPIRE].

[33] J.F. Donoghue, Leading quantum correction to the Newtonian potential, Phys. Rev. Lett. 72 (1994) 2996 [gr-qc/9310024] [INSPIRE].

[34] J.F. Donoghue, General relativity as an effective field theory: The leading quantum corrections, Phys. Rev. D 50 (1994) 3874 [gr-qc/9405057] [INSPIRE].

[35] J.F. Donoghue, M.M. Ivanov and A. Shkerin, EPFL Lectures on General Relativity as a Quantum Field Theory, arXiv:1702.00319 [INSPIRE].

[36] J. Donoghue, Quantum gravity as a low energy effective field theory, Scholarpedia 12 (2017) 32997 [INSPIRE]. 
[37] J. Ellis, J.R. Espinosa, G.F. Giudice, A. Hoecker and A. Riotto, The Probable Fate of the Standard Model, Phys. Lett. B 679 (2009) 369 [arXiv:0906.0954] [InSPIRE].

[38] J. Elias-Miro, J.R. Espinosa, G.F. Giudice, H.M. Lee and A. Strumia, Stabilization of the Electroweak Vacuum by a Scalar Threshold Effect, JHEP 06 (2012) 031 [arXiv:1203.0237] [INSPIRE].

[39] D. Boyanovsky, Condensates and quasiparticles in inflationary cosmology: mass generation and decay widths, Phys. Rev. D 85 (2012) 123525 [arXiv:1203.3903] [INSPIRE].

[40] J. Lankinen and I. Vilja, Decaying massive particles in the matter and radiation dominated eras, Phys. Rev. D 97 (2018) 065004 [arXiv:1801.03757] [inSPIRE].

[41] R. Erdem and K. Gültekin, A mechanism for formation of Bose-Einstein condensation in cosmology, arXiv:1908.08784 [INSPIRE]. 